ПРИЛОЗИ, Одделение за природно-математички и биотехнички науки, МАНУ, том 36, бр.2, стр. 153-164 (2015)

CONTRIBUTIONS, Section of Natural, Mathematical and Biotechnical Sciences, MASA, Vol. 36, No. 2, pp. $153-164$ (2015)

Received: April 24, 2015

ISSN 1857-9027

Accepted: October 6, 2015

UDC:515.142:514.548

Original scientific paper

\title{
SOME WEAKER FORMS OF SMOOTH FUZZY CONTINUOUS FUNCTIONS
}

\author{
Chandran Kalaivani ${ }^{1}$, Rajakumar Roopkumar ${ }^{2 *}$ \\ ${ }^{1}$ Department of Mathematics, SSN College of Engineering, Kalavakkam - 603 110, India \\ ${ }^{2}$ Department of Mathematics, Alagappa University, Karaikudi - 630 004, India \\ ${ }^{*}$ Corresponding author, email: roopkumarr@ rediffmail.com
}

\begin{abstract}
In this paper we introduce various notions of continuous fuzzy proper functions by using the existing notions of fuzzy closure and fuzzy interior operators like $R_{\tau}^{r}$-closure, $R_{\tau}^{r}$-interior, etc., and present all possible relations among these types of continuities. Next, we introduce the concepts of $\alpha$-quasi-coincidence, $q_{\alpha}^{r}$-pre-neighborhood, $q_{\alpha}^{r}$-pre-closure and $q_{\alpha}^{r}$ - pre-continuous function in smooth fuzzy topological spaces and investigate the equivalent conditions of $q_{\alpha}^{r}$ - pre-continuity.
\end{abstract}

Key words: fuzzy proper function; smooth fuzzy topology; smooth fuzzy continuity; fuzzy closure; fuzzy interior

\section{INTRODUCTION}

Šostak [28] defined $I$-fuzzy topology as an extension of Chang's fuzzy topology [2]. It has been developed in many directions by many authors. For example see $[8,16]$. Ramadan [23] gave a similar definition of fuzzy topology on a fuzzy set in Šostak's sense and called by the name "smooth fuzzy topological space".

On the other hand, studying different forms of continuous functions in topological space is an interesting area of research which attracts many researchers. In the fuzzy context, after the introduction of fuzzy proper function from a fuzzy set in to a fuzzy set [1], several notions of continuous fuzzy proper functions between Chang's fuzzy topological spaces are defined and their properties are discussed in [3]. The concepts of smooth fuzzy continuity, weakly smooth fuzzy continuity, $q n$-weakly smooth fuzzy continuity, $(\alpha, \beta)$-weakly smooth fuzzy continuity of a fuzzy proper function on smooth fuzzy topological spaces and their inter-relations are investigated in [5, $23,26,27,10]$.

Lee and Lee [19] introduced the notion of fuzzy $r$-interior which is an extension of Chang's fuzzy interior. Using fuzzy $r$-interior, they define fuzzy $r$-semiopen sets and fuzzy $r$-semicontinuous maps which generalize fuzzy semiopen sets and fuzzy semicontinuous maps in Chang's fuzzy topology, respectively. Some basic properties of fuzzy $r$ semiopen sets and fuzzy $r$-semicontinuous maps are investigated in [19]. In [22], the concepts of several types of weak smooth compactness are introduced and investigated some of their properties.

In $[7,20]$, the notions of fuzzy semicontinuity, fuzzy $\gamma$-continuity of a fuzzy proper functions, fuzzy separation axioms, fuzzy connectedness and fuzzy compactness are defined.

Ganguly and Saha [6] introduced the notions of $\delta$-cluster points and $\theta$-cluster points in Chang's fuzzy topological spaces. Kim and Park [15] introduced $\delta$ closure in Šostak's fuzzy topological spaces. Kim and Ko [13] introduced fuzzy super continuity, fuzzy $\delta$ continuity, fuzzy almost continuity in the context of Šostak's fuzzy topological spaces. They proved that fuzzy super continuity implies both fuzzy $\delta$-continuity and fuzzy almost continuity. Similar works are discussed by various researchers, see $[12,14,18,21]$.

By using the existing notions of fuzzy closure and fuzzy interior operators, we introduce the concepts of fuzzy weakly $\delta$-continuity, fuzzy weakly $\delta$ $r_{1}$-continuity, fuzzy weakly $\delta$-[r,q]$]_{1}$-continuity, 
fuzzy weakly $\delta$ - $r_{2}$-continuity, fuzzy weakly $\delta$ - $[r, q]_{2}-$ continuity, fuzzy weakly $\delta$ - $r_{3}$-continuity, fuzzy weakly $\delta$ - $r_{4}$-continuity, fuzzy almost $r_{1}$-continuity, fuzzy almost $[r, q]_{1}$ - continuity, fuzzy almost $r_{2}$-continuity, fuzzy almost $[r, q]_{2}$-continuity, fuzzy almost $r_{3}$-continuity and fuzzy almost $r_{4}$-continuity and discuss the inter-relations among them.

Further, by introducing the notions $\alpha$-quasicoincidence, $q_{\alpha}^{r}$-pre-neighborhood, $q_{\alpha}^{r}$-pre-closure and $q_{\alpha}^{r}$-pre-continuity, we investigate the relations between $q_{\alpha}^{r}$ - pre-continuity and the property $F\left(P C l_{a}(A, r)\right) \leq P C l_{a}(F(A), r)$, for every $A \leq \mu$ in smooth fuzzy topological spaces.

\section{PRELIMINARIES}

Let $X, S$ be non-empty sets. We denote by $I$, $I_{0}, I^{X}, 0_{X}, \mu$ and $v$ respectively the unit interval $[0$, $1]$, the interval $[0,1]$, the set of all fuzzy subsets of $X$, the zero function on $X$, a fixed fuzzy subset of $X$ and a fixed fuzzy subset of $S$. For $X=\left\{x_{1}, x_{2}, \ldots\right.$, $\left.x_{n}\right\}$ and $\lambda_{\mathrm{i}} \in I, i \in\{1,2, \ldots, n\}$, we denote the fuzzy subset $\mu$ of $X$ which maps $x_{i}$ to $\lambda_{\mathrm{i}}$ for every $i=$ $1,2, \ldots, n$ by $\mu \frac{\left[\lambda_{1}, \lambda_{2}, \ldots, \lambda_{n}\right]}{\left[x_{1}, x_{2}, \ldots, x_{n}\right]}$. A fuzzy point [15] in $X$ is defined by $P_{x}^{\lambda}(t)=\left\{\begin{array}{lll}\lambda & \text { if } & t=x \\ 0 & \text { if } & t \neq x\end{array}\right.$, where $0<\lambda \leq 1$. By $P_{x}^{\lambda} \in \mu$ we mean that $\lambda \leq \mu(x)$.

Definition 1 [23]: A smooth fuzzy topology on a fuzzy set $\mu \in I^{X}$ is a map $\tau: \mathcal{J}_{\mu}=\left\{U \in I^{X}: U \leq\right.$ $\mu\} \rightarrow I$, satisfying the following axioms:

1. $\tau\left(0_{X}\right)=\tau(\mu)=1$

2. $\tau\left(A_{1} \Lambda A_{2}\right) \geq \tau\left(A_{1}\right) \Lambda \tau\left(A_{2}\right), \forall A_{1}, A_{2} \in \mathcal{J}_{\mu}$,

3. $\tau\left(\bigvee_{i \in \Gamma} A_{i}\right) \geq \Lambda_{i \in \Gamma} \tau\left(A_{i}\right)$ for every family $\left(A_{i}\right)_{i \in \Gamma} \subseteq \mathcal{J}_{\mu}$

The pair $(\mu, \tau)$ is called a smooth fuzzy topological space.

A fuzzy subset $U \in \mathcal{J}_{\mu}$ is said to be fuzzy open if $\tau(U)>0$ and fuzzy closed if $\tau(\mu-U)>0$.

Definition 2 [1]: Let $U, V \in \mathcal{J}_{\mu}$ are said to be quasicoincident referred to $\mu$ (written as $U q V[\mu]$ ) if there exists $x \in X$ such that $U(x)+V(x)>\mu(x)$. If $U$ is not quasi-coincident with $V$, then we write, $U \bar{q} V[\mu]$.

A fuzzy set $U \in \mathcal{J}_{\mu}$ is called a $q$-neighborhood of a fuzzy point $P_{x}^{\lambda}$ in $\mu$ if $P_{x}^{\lambda} q U[\mu]$ and $\tau(U)$ $>0$.

Definition 3[1]: Let $\mu \in I^{X}$ and $v \in I^{S}$. A non-zero fuzzy subset $F$ of $X \in S$ is said to be a fuzzy proper function from $\mu$ to $v$ if

1. $F(x, s) \leq \min \{\mu(x), v(s)\}, \forall(x, s) \in X \times S$,
2. for each $x \in X$ with $\mu(x)>0$, there exists a unique $s_{0} \in S$ such that $F\left(x, s_{0}\right)=\mu(x)$ and $F(x, s)=0$ if $s \neq s_{0}$

Definition 4 [1]: Let $F$ be a fuzzy proper function from $\mu$ to $v$. If $U \in \mathcal{J}_{\mu}$ and $V \in \mathcal{J}_{\mu}$, then $F(U): S \rightarrow I$ and $F^{-1}(V): X \rightarrow I$ are defined by

$$
\begin{aligned}
& (F(U))(s)=\sup \{F(x, s) \Lambda U(x): x \in X\}, \forall s \in S, \\
& \left(F^{-1}(V)\right)(x)=\sup \{F(x, s) \Lambda V(s): s \in S\}, \forall x \in X .
\end{aligned}
$$

The inverse image of a fuzzy subset $V$ under a fuzzy proper function $F$ can be easily obtained as $\left(F^{-}\right.$ $\left.{ }^{l}(V)\right)(x)=\mu(x) \Lambda V(s)$, where $s \in S$ is the unique element such that $F(x, s)=\mu(x)$.

Definition 5 [5]: A fuzzy proper function $F: \mu \rightarrow v$ is said to be injective (or one-to-one) if $F\left(x_{1}, s\right)>0$ and $F\left(x_{2}, s\right)>0$, for some $x_{1}, x_{2} \in X$ and $s \in S$, then $x_{1}=$ $x_{2}$.

Definition 6 [4]: Let $(\mu, \tau)$ be a smooth fuzzy topological space. For $r \in I_{0}, A \in \mathcal{J}_{\mu}$,

- $C_{\tau}: \mathcal{J}_{\mu} \times I_{0} \rightarrow \mathcal{J}_{\mu}$ is defined by $C_{\tau}(A, r)=$ $\Lambda\left\{K \in \mathcal{J}_{\mu}: A \leq K, \tau(\mu-K) \geq r\right\}$,

- $I_{\tau}: \mathcal{J}_{\mu} \times I_{0} \rightarrow \mathcal{J}_{\mu}$ is defined by $I_{\tau}(A, r)=$ $\vee\left\{S \in \mathcal{J}_{\mu}: S \leq A, \tau(S) \geq r\right\}$.

Definition 7 (Cf. [18]): Let $(\mu, \tau)$ be smooth fuzzy topological space, $U \in \mathcal{J}_{\mu}$, and $r \in I_{0}$. Then

- $U$ is called fuzzy r-preopen if $U \leq I_{\tau}\left(C_{\tau}(U, r), r\right)$,

- $U$ is called fuzzy r-preclosed if $U \geq$ $C_{\tau}\left(I_{\tau}(U, r), r\right)$

Definition 8 [13]: Let $(\mu, \tau)$ be a smooth fuzzy topological space and let $A \in \mathcal{J}_{\mu}, r \in I_{0}$. Then,

- $A$ is called a $Q_{\tau}^{r}$-neighborhood of $P_{x}^{\lambda}$ if $P_{x}^{\lambda} q A[\mu]$ with $\tau(A) \geq r$,

- $A$ is called a $R_{\tau}^{r}$-neighborhood of $P_{x}^{\lambda}$ if $P_{x}^{\lambda} q A[\mu]$ with $A=I_{\tau}\left(C_{\tau}(A, r), r\right)$.

Definition 9 [11]: Let $(\mu, \tau)$ be a smooth fuzzy topological space and let $A \in \mathcal{J}_{\mu}, r \in I_{0}$. Then, we define,

- Smooth fuzzy $R_{\tau}^{r}$-closure of A by

$\mathbb{D}_{\tau}(A, r)=\bigvee\left\{P_{x}^{\lambda} \in \mu: C_{\tau}(U, r) q A[\mu], \forall R_{\tau}^{r}-n e i g h-\right.$ borhood $U$ of $\left.P_{x}^{\lambda}\right\}$.

- Smooth fuzzy $R_{\tau}^{r}$-interior of A by

$\mathbb{I}_{\tau}(A, r)=\mathrm{V}\left\{K \in \mathcal{J}_{\mu}: A \geq C_{\tau}(K, r), K=\right.$ $\left.I_{\tau}\left(C_{\tau}(K, r), r\right)\right\}$.

Theorem 1 [11]: Let $(\mu, \tau)$ be a smooth fuzzy topological space. For $A \in \mathcal{J}_{\mu}$ and $r \in I_{0}$, then

$$
\begin{gathered}
\mathbb{D} \tau(A, r) \wedge\left\{K \in \mathcal{J}_{\mu}: A \leq I_{\tau}(K, r), K=\right. \\
\left.C_{\tau}\left(I_{\tau}(K, r), r\right)\right\} .
\end{gathered}
$$


Definition 10 (Cf. [13]): Let $(\mu, \tau)$ and $(\nu, \sigma)$ be two smooth fuzzy topological spaces and $F: \mu \rightarrow v$ be a fuzzy proper function. Then, $F$ is called fuzzy almost continuous or FAC if for every $R_{\sigma}^{r}$-neighborhood $V$ of $F\left(P_{x}^{\lambda}\right)$, there exists an $Q_{\tau}^{r}$-neighborhood $U$ of $P_{x}^{\lambda}$ such that $F(U) \leq V$.

Theorem 2 [9]: Let $F: \mu \rightarrow v$ be a fuzzy proper function such that $v=F(\mu)$. If $F$ is one-to-one, then $F^{-1}(v$ $-V)=\mu-F^{-1}(V), \forall V \in \mathcal{J}_{\mu}$.

\section{FUZZY WEAKLY $\delta$-CONTINUOUS AND FUZZY ALMOST CONTINUOUS FUNCTIONS}

Definition 11: Let $(\mu, \tau)$ and $(v, \sigma)$ be smooth fuzzy topological spaces, $F: \mu \rightarrow$ vbe a fuzzy proper function and $r, q \in I_{0}$ be fixed. Then, $F$ is called

(1) fuzzy weakly $\delta$-continuous or $F W \delta$-C if for every $R_{\sigma}^{r}$-neighborhood $V$ of $F\left(P_{x}^{\lambda}\right)$, there exists an $R_{\tau}^{r}$-neighborhood $U$ of $P_{x}^{\lambda}$ such that $F(C \tau(U, r)) \leq$ $V$,

(2) fuzzy weakly $\delta$ - $r_{1}$-continuous or $F W \delta-r_{1}$-C if $F\left(\mathbb{D}_{\tau}(A, r)\right) \leq \mathbb{D} \sigma(F(A), r), \forall A \in \mathcal{J}_{\mu}$,

(3) fuzzy weakly $\delta$ - $[r, q]_{1}$-continuityor $F W \delta-[r, q]_{1}-C$ if

$F\left(\mathbb{D}_{\tau}(A, r)\right) \leq \mathbb{D} \sigma(F(A), q), \forall A \in \mathcal{J}_{\mu}$,

(4) fuzzy weakly $\delta$ - $r_{2}$-continuous or $\mathrm{FW} \delta-r_{2}-C$ if

$\mathbb{D}_{\tau}\left(F^{-1}(V), r\right) \leq F^{-1}\left(\mathbb{D}_{\sigma}(V, r)\right), \forall V \in$,

(5) fuzzy weakly $\delta$ - $[r, q]_{2}$-continuous or $F W \delta$ - $[r, q]_{1}-$ $C$ if

$$
\mathbb{D}_{\tau}\left(F^{-1}(V), r\right) \leq F^{-1}\left(\mathbb{D}_{\sigma}(V, q)\right), \forall V \in \mathcal{J}_{\mu},
$$

(6) fuzzy weakly $\delta$ - $r_{3}$-continuous or $F W \delta-r_{3}-C$ if $\mathbb{D}_{\tau}\left(F^{-1}(V), r\right)=F^{-1}(V), \forall V \in \mathcal{J}_{\mu}$ with $V=\mathbb{D}_{\sigma}(V, r)$,

(7) fuzzy weakly $\delta$-r $r_{4}$-continuous or $F W \delta-r_{4}-C$ if $\mathbb{D}_{\tau}\left(\mu-F^{-1}(V), r\right)=\mu-F^{-1}(V), \forall V \in \mathcal{J}_{\mu}$ with $V=$ $\mathbb{I}_{\sigma}(V, r)$.

Theorem 3 Let $F:(\mu, \tau) \rightarrow(\nu, \sigma)$ be a one-to-one fuzzy proper function with $v=F(\mu)$. If $F$ is fuzzy weakly $\delta$-continuous, then $F$ is fuzzy weakly $\delta$ - $r_{1}$ continuous

Proof. Suppose that there exist $\mathrm{A} \in \mathcal{J}_{\mu}$ and $r \in I_{0}$ such that

$$
\mathrm{F}\left(\mathbb{D}_{\tau}(A, r)\right)(s)>\mathbb{D}_{\sigma}(F(A), r)(s),
$$

for some $s \in S$. Then, there exists $x \in X$ such that $F(x, s)>0$. Since $F$ is one-to-one and $F(\mu)=v$, we have $\left.F\left(\mathbb{D}_{\tau}(A, \quad r)\right)(s)=\mathbb{D}_{\tau}(A, \quad r)\right)(x)>$ $\mathbb{D}_{\sigma}(F(A), r)(s)$. Now we choose a real number $\eta$ such that $\left.\mathbb{D}_{\tau}(A, r)\right)(x)>\eta>\mathbb{D}_{\sigma}(F(A), r)(s)$. Since $P_{S}^{\eta} \notin \mathbb{D}_{\sigma}(F(A), r)$, there exists an $R_{\sigma}^{r}$-neighborhood $V$ of $\mathrm{F}\left(P_{x}^{\eta}\right)=P_{s}^{\eta}$ such that $C_{\tau}(V, r) \bar{q} F(A)[V]$ which implies that $\mathrm{F}(\mathrm{A}) \leq v-C_{\tau}(V, r)$. Since $F$ is fuzzy weakly $\delta$-continuous, there exists an $R_{\tau}^{r}$ neighborhood $U$ of $P_{x}^{\eta}$ such that $F\left(C_{\tau}(U, r) \leq V \leq\right.$ $C_{\tau}(V, r)$. Thus, $F(\mathrm{~A}) \leq v-F\left(C_{\tau}(U, r)\right)$. Using the facts that $F$ is one-to-one and $F(\mu)=v$ and using Theorem 2 , we get

$$
\begin{aligned}
A \leq F^{-1}(F(A)) & \leq F^{-1}\left(v-F\left(C_{\tau}(U, r)\right)\right) \\
=\mu & -F^{-1}\left(F\left(C_{\tau}(U, r)\right)\right) \leq \mu-C_{\tau}(U, r) .
\end{aligned}
$$

Therefore, $\mathrm{A} \bar{q} C_{\tau}(U, r)[\mu]$ and $\left.P_{x}^{\eta} \notin \mathbb{D}_{\tau}(A), r\right)$ which implies that $P_{s}^{\eta}=F\left(P_{x}^{\eta}\right) \notin F\left(\mathbb{D}_{\tau}(A, r)\right)$, which is a contradiction to $F\left(\mathbb{D}_{\tau}(A, r)\right)>\eta$. Hence, it follows that $F\left(\mathbb{D}_{\tau}(A, r)\right) \leq \mathbb{D}_{\sigma}(F(A), r)$.

The statement of the above theorem is not true when $F$ is not one-to-one or $F(\mu) \neq v$. The following counterexamples justify our statement.

Counterexample 1: Let $X=\{x, y\}, S=\{s, t\}, \mu\left\{\begin{array}{c}{[0.8,0.7]} \\ {[x, y]}\end{array} \in\right.$ $I^{X}, v_{[s, t]}^{[0.8,0]} \in I^{S}, U_{1}{ }_{[x, y]}^{[0.4,0.3]} \in \mathcal{J}_{\mu}$ and $V_{1}{ }_{[0, t]}^{[0.4,0]} \in \mathcal{J}_{\nu}$.

We define $\tau: \mathcal{J}_{\mu} \rightarrow I$ and $\sigma: \mathcal{J}_{\nu} \rightarrow I$ by

$\tau(U)=\left\{\begin{array}{cc}1, & U=0_{X}, \text { or } \mu \\ 0.7, \quad U=U_{1}, \\ 0, \quad \text { otherwise }\end{array}\right.$

and

$\sigma(V)=\left\{\begin{array}{c}1, \quad V=0_{S} \text { or } v \\ 0.6, \quad V=V_{1}, \\ 0, \quad \text { otherwise. }\end{array}\right.$

If the fuzzy proper function $F:(\mu, \tau) \rightarrow$ $(\nu, \sigma)$ is defined by

$$
F(x, s)=0.8, F(x, t)=0, F(y, s)=0.7, F(y, t)=0,
$$

Then $F$ is not one-to-one and $F(\mu)_{[s, t]}^{[0.8,0]}=v$. We fix $r=0.5$. For $P_{l}^{\eta} \in \mu$ and for the $R_{\sigma}^{r}$-neighborhood $V_{1}$ of $F\left(P_{l}^{\eta}\right)$, we can choose $U_{1}$ as an $R_{\tau}^{r}$-neighborhood of $P_{l}^{\lambda}$ satisfying $F\left(C_{\tau}\left(U_{1}, r\right)\right)_{[s, t]}^{[0.4,0]}=V_{1}$. For $v$ we find $\mu$ such that $F\left(C_{\tau}(\mu, r)\right)=v$. Thus $F$ is fuzzy weakly $\delta$-continuous.

Consider the fuzzy point $P_{y}^{0.45} \in \mu$ and the fuzzy set $A_{[x, y]}^{[0.04]} \in \mathcal{J}_{\mu}$. If $U \in \mathcal{J}_{\mu}$ is such that $U$ $=I_{\tau}\left(C_{\tau}(U, r), r\right)$, then $U=0_{X}$ or $\mu$ or $U_{1}$ and both are the $R_{\tau}^{r}$ ods $P_{y}^{0.45}$. Here, $C_{\tau}\left(U_{1}, r\right), q A[\mu]$ and $C_{\tau}(\mu, r), q A[\mu]$. Therefore, $P_{y}^{0.45} \in \mathbb{D}_{\tau}(A, r)$ and $F\left(P_{y}^{0.45}\right)=P_{s}^{0.45} \in F\left(\mathbb{D}_{\tau}(A, r)\right.$. Since, $V_{1}(s)+$ $0.45>0.8=v(s) \quad$ and $\quad I_{\sigma}\left(C_{\sigma}\left(V_{1}, r\right), r\right)=$ $C_{\sigma}\left(\left(v-V_{1}\right)_{[s, t]}^{[0.4,0]}, r\right)=V_{1}$ 
$V_{1}$ is $R_{\tau}^{r}$-neighborhood of $P_{S}^{0.45}$. We note that $F(\mathrm{~A})^{[0.4,0]}{ }_{[s, t]}^{[0}$ and $F(A) \bar{q} C_{\sigma}(V, r)[v]$ and hence $P_{S}^{0.45} \notin \mathbb{D}_{\sigma}(F(A), r)$. Therefore $F$ is not fuzzy weakly $\delta$ - $r_{1}$-continuous.

Counterexample 2: Let $X=\{x, y\}, S=\{s, t\}$, $\mu\left\{\begin{array}{c}{[0.9,0.8]} \\ x, y\end{array} \in I^{X}, v_{[s, t]}^{[1,1,]} \in I^{S}, U_{1}{ }_{[x, y]}^{[0.4,0.3]} \in \mathcal{J}_{\mu} \quad\right.$ and $V_{1} \underset{[s, t]}{[0.5,0.5]} \in \mathcal{J}_{v}$.

We define $\tau: \mathcal{J}_{\mu} \rightarrow$ Iand $\sigma: \mathcal{J}_{\nu} \rightarrow I$ by

$\tau(U)= \begin{cases}1, & U=0_{X} \text { or } \mu \\ 0.6, & U=U_{1} \\ 0, & \text { otherwise }\end{cases}$

and

$\sigma(V)= \begin{cases}1, & V=0_{S} \text { or } v \\ 0.5, & V=V_{1}, \\ 0, & \text { otherwise. }\end{cases}$

Let the fuzzy proper function $F:(\mu, \tau) \rightarrow(\nu, \sigma)$ be defined by

$$
F(x, s)=0.9, F(x, t)=0, F(y, s)=0, F(y, t)=0.8 .
$$

Then, $F(\mu)_{[s, t]}^{[0.9,0.8]} \neq v$. If $r=0.5$, and for the $R_{\sigma^{-}}^{r}$ neighborhood $V_{1}$ of $F\left(P_{l}^{\eta}\right)$, we can fine $U_{1}$ as a required $R_{\tau}^{r}$-neighborhood of $P_{l}^{\eta} \in \mu$. Indeed, we first note that $F\left(C_{\tau}\left(U_{1}, r\right)\right)^{[0.5,0.5]}=\mathrm{V}_{1}$. Since the only $R_{\sigma}^{r}$-neighborhoods of $F\left(P_{l}^{\eta}\right)$ are $V_{1}$ and $v$, it follows that $F$ is fuzzy weakly $\delta$-continuous.

Consider $P_{y}^{0.55} \in \mu$ and $A_{[x, y]}^{[0.04]} \in \mathcal{J}_{\mu}$. Since $P_{y}^{0.55} q U_{1}[\mu]$ and $P_{y}^{0.55} q \mu[\mu], U_{1}$ and $\mu$ are the $R_{\tau}^{r}$-neighborhoods of $P_{y}^{0.55}$. Since $C_{\tau}\left(U_{1}, r\right), q A[\mu]$ and $C_{\tau}(\mu, r), q A[\mu]$, we have $P_{y}^{0.55} \in \mathbb{D}_{\tau}(A, r) \quad$ and $\quad F\left(P_{y}^{0.55}\right)=P_{t}^{0.55} \in$ $\mathbb{D}_{\tau}(A, r)$. Using

$V_{1}(t)+0.55>1=v(t)$ and

$I_{\sigma}\left(C_{\sigma}\left(V_{1}, r\right), r\right)=I_{\sigma}\left(\left(v-V_{1}\right)_{[s, t]}^{[0.5,0.5]}, r\right)=V_{1}$,

we get that $V_{1}$ is an $R_{\tau}^{r}$-neighborhood of $P_{t}^{0.55}$. But $F \quad(A){ }_{[s, t]}^{[0.4,0]} \bar{q} C_{\sigma}(V, r)[v]$ implies that $P_{t}^{0.55} \notin$ $\mathbb{D}_{\sigma}(F(A), r)$.

Theorem 4: Let $F:(\mu, \tau) \rightarrow(\nu, \sigma)$ be a fuzzy properfunction. If (a) $F$ is fuzzy weakly $\delta-r_{1}$-continuous, (b) $F$ is fuzzy weakly $\delta$ - $r_{2}$-continuous, (c) $F$ is fuzzy weakly $\delta$ - $r_{3}$-continuous, then $\left.(a) \Rightarrow b\right) \Rightarrow(c)$.

Proof is straightforward.

Theorem 5: Let $F:(\mu, \tau) \rightarrow(\nu, \sigma)$ be a one-to-one fuzzy proper function with $v=F(\mu)$. If $F$ is fuzzy weakly $\delta$ - $r_{3}$-continuous, then $F$ is $\delta$ - $r_{4}$-continuous.

Proof. Let $V \in \mathcal{J}_{v}$ with $V=\mathbb{I}_{\sigma}(V, r)$. Then,
$v-V=v-\mathbb{I}_{\sigma}(V, r)=\mathbb{D}_{\sigma}(v-V, r)$. By using the hypothesis, we get $\mathbb{D}_{\tau}\left(\mathrm{F}^{-1}(v-V), r\right)=\mathrm{F}^{-1}(v-V)$. Since $F$ is one-to-one and $v=F(\mu)$ and by Theorem 2 , we have $F^{-1}(v-V)=\mu-F^{-1}(V)$. Therefore, $\mathbb{D}_{\tau}\left(\mu-F^{-1}(V), r\right)=\mu-F^{-1}(V)$.

The statement of the above theorem is not true when $F$ is not one-to-one or $F(\mu) \neq v$. The following counterexamples justify our statement.

Counterexample 3: Let $X=\{x, y\}, S=\{s, t\}$,. We define

$\mu\left\{\begin{array}{c}{[0.8,0.6]} \\ {[x, y]}\end{array} \in I^{X}, v^{[0.8,0]} \in I^{S}, U_{n} \begin{array}{c}{\left[0.4+\frac{1}{n+10}, 0.4+\frac{1}{n+10}\right]} \\ {[x, y]}\end{array}\right.$, where $n=1,2, \ldots$ and $V_{1}{ }_{[s, t]}^{[0.4,0]} \in \mathcal{J}_{\nu}$. If $\tau: \mathcal{J}_{\mu} \rightarrow I$ and $\sigma: \mathcal{J}_{v} \rightarrow I$ are defined by

$\tau(U)= \begin{cases}1, & U=0_{X} \text { or } \mu \\ 0.6, & U=U_{n} \forall n \text { or } \vee U_{n}, \\ 0, & \text { otherwise }\end{cases}$

and

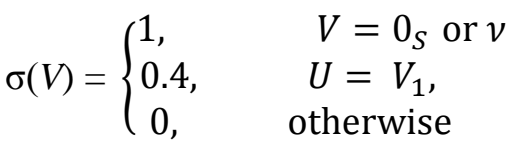

then $(\mu, \tau)$ and $(\nu, \sigma)$ are smooth fuzzy topological spaces. Let the fuzzy proper function $F:(\mu, \tau) \rightarrow$ $(v, \sigma)$ be defined by

$$
F(x, s)=0.8, F(x, t)=0, F(y, s)=0.6, F(y, t)=0 .
$$

We fix $r=0.4$. Since $C_{\sigma}\left(V_{1}, r\right)=V_{1}=$ $I_{\sigma}\left(V_{1}, r\right)$ and $C_{\tau}\left(U_{n}, r\right)=U_{n}=I_{\tau}\left(U_{n}, r\right), n=1$, 2 , ..., we get $\mathbb{D}_{\sigma}\left(V_{1}, r\right)=V_{1}, F^{-1}\left(V_{1}\right)_{x, y}^{[0.4,0.4]} \leq$ $I_{\tau}\left(U_{n}, r\right)$, and $C_{\tau}\left(I_{\tau}\left(U_{n}, r\right), r\right)=U_{n}$. Therefore, $\mathbb{D}_{\tau}\left(F^{-1}\left(V_{1}\right), r\right)=\left(\wedge U_{n}\right)_{[x, y]}^{[0.4,0.4]}=F^{-1}\left(V_{1}\right)$ and hence $F$ is fuzzy weakly $\delta-r_{3}$-continuous.

We note that $I_{\sigma}\left(V_{1}, r\right)=V_{1}$ and $\mathbb{D}_{\tau}\left(\mu-F^{-}\right.$ $\left.\left.{ }^{1}\left(V_{1}\right)\right)_{[x, y]}^{[0.4,0.2]}, r\right)=\wedge U_{n} \neq \mu-F^{-1}\left(V_{1}\right)$. Thus, $F$ is not fuzzy weakly $\delta-r_{4}$-continuous.

Counterexample 4: Let $\mathrm{X}=\{x, y\}, \mathrm{S}=\{s, t\}$. Define the fuzzy subsets $\mu\left\{\begin{array}{c}{[0.8,0.6]} \\ x, y\end{array} \in I^{X}, v_{[s, t]}^{[0.8,0.8]} \in\right.$ $I^{S}, U_{n} \underset{\left[0.4+\frac{1}{n+10^{\prime}}, 0.4+\frac{1}{n+10}\right]}{[x, y]}$, where $n=1,2, \ldots$ and $V_{1}{ }_{[s, t]}^{[0.4,0.4]}$. Let $\tau: \mathcal{J}_{\mu} \rightarrow I$ and $\sigma: \mathcal{J}_{\nu} \rightarrow I$ be defined by

$\tau(U)=\left\{\begin{array}{r}1, \quad U=0_{X} \text { or } \mu \\ 0.6, \quad \begin{array}{c}U=U_{n} \forall n \text { or } \vee U_{n}, \\ 0,\end{array} \text { otherwise }\end{array}\right.$

and

$\sigma(V)=\left\{\begin{array}{lc}1, & V=0_{S} \text { or } v \\ 0.5, & U=V_{1}, \\ 0, & \text { otherwise }\end{array}\right.$

If $F:(\mu, \tau) \rightarrow(\nu, \sigma)$ is defined by 


$$
F(x, s)=0.8, F(x, t)=0, F(y, s)=0, F(y, t)=0,
$$

Then $F$ is one-to-one and $F(\mu){ }_{[s, t]}^{[0.8,0.6]} \neq v$. We fix $r=0.5$. From $C_{\sigma}\left(V_{1}, r\right)=V_{1}=I_{\sigma}\left(V_{1}, r\right)=V_{1}$, we have $\mathbb{D}_{\sigma}\left(V_{1}, r\right)=V_{1}$. Since $C_{\tau}\left(U_{n}, r\right)=U_{n}=$ $I_{\tau}\left(U_{n}, r\right), n=1,2, \ldots$, we get that

and

$$
\mathrm{F}^{-1}\left(V_{1}\right) \underset{x, y}{[0.4,0.4]} \leq I_{\tau}\left(U_{n}, r\right)
$$

$$
C_{\tau}\left(I_{\tau}\left(U_{n}, r\right), r\right)=U_{n} .
$$

Therefore, $\left.\quad \mathbb{D}_{\tau} \quad\left(F^{-1}\left(V_{1}\right), r\right)\right)=$ $\left(\wedge U_{n}\right)^{[0.4,0.4]}{ }_{[x, y]}^{[-1}=F^{-1}\left(V_{1}\right)$ and hence $F$ is fuzzy weakly $\delta-r_{3}$-continuous. From the observations, $I_{\sigma}\left(V_{1}, r\right)=V_{1}$ and $\left.\mathbb{D}_{\tau}\left(\mu-F^{-1}\left(V_{1}\right)\right), r\right)=$ $\wedge U_{n} \neq \mu-F^{-1}\left(V_{1}\right)$, we conclude that $F$ is not fuzzy weakly $\delta$-r $r_{4}$-continuous.

The following counterexample shows that fuzzy weakly $\delta-r_{4}$-continuous function is not a fuzzy weakly $\delta$-continuous function.

Counterexample 5: Let $X=\{x, y\}, S=\{s, t\}$. Define $\mu\left\{\begin{array}{c}{[0.8,0.7]} \\ {[x, y]}\end{array} \in I^{X}, v_{[s, t]}^{[0.8,0.7]} \in I^{S}\right.$ and $V_{1}{ }_{[s, t]}^{[0.4,0.3]} \in$ $\mathcal{J}_{v}$

If $\tau: \mathcal{J}_{\mu} \rightarrow I$ and $\sigma: \mathcal{J}_{\nu} \rightarrow I$ are defined by

$\tau(U)=\left\{\begin{array}{lr}1, & U=0_{X} \text { or } \mu \\ 0, & \text { otherwise }\end{array}\right.$

and

$\sigma(V)= \begin{cases}1, & V=0_{S} \text { or } v \\ 0.5, & V=V_{1}, \\ 0, & \text { otherwise }\end{cases}$

then $(\mu, \tau)$ and $(\nu, \sigma)$ are smooth fuzzy topological spaces. Let the fuzzy proper function $F:(\mu, \tau) \rightarrow$ $(v, \sigma)$ be defined by

$$
F(x, s)=0.8, F(x, t)=0, F(y, s)=0, F(y, t)=0.7 .
$$

Fix $r=0.5$. If $I_{\sigma}\left(C_{\sigma}(V, r), r\right)=V$, then $V=0_{S}$ or $V=v$ or $V=V_{1}$. But $C_{\sigma}\left(\left(v-V_{1}\right)_{[0, t]}^{[0.4,0.4]}, r\right) \$ V_{1}$ implies that $\mathbb{I}_{\sigma}\left(V_{1}, r\right)=0_{S}$. Since $\mathbb{D}_{\tau}\left(\mu-F^{-1}(V), \mathrm{r}\right)$ $=\mu-F^{-1}(V)$, for every $V$ with $\mathbb{I}_{\sigma}(V, r)=V$, we get that $F$ is fuzzy weakly $\delta$ - $r_{4}$-continuous.

Next, we claim that $F$ is not fuzzy weakly $\delta$ continuous. Since $F\left(P_{y}^{0.45}\right)=P_{t}^{0.45} q V_{1}[v]$ and $I_{\sigma}\left(C_{\sigma}(V, r), r\right)=V_{1}, V_{1}$ is an $R_{\sigma}^{r}$-neighborhood of $P_{t}^{0.45}$. The only $R_{\tau}^{r}$-neighborhood of $P_{y}^{0.45}$ is $\mu$, for which we have $F\left(C_{\tau}(\mu, r)\right)=F(\mu) \$ V_{1}$. Hence, our claim holds. forward.

The proof of the following theorem is straight-

Theorem 6: Let $r, q \in I_{0}$ and $F:(\mu, \tau) \rightarrow(\nu, \sigma)$.
1. If $r<q$ and if $F$ is fuzzy weakly $\delta$ - $r_{1}$-continuous, then $F$ is fuzzy weakly $\delta$ - $[r, q]_{1}$-continuous.

2. If $q<r$ and if $F:(\mu, \tau) \rightarrow(v, \sigma)$ is fuzzy weakly $\delta$ - $[r, q]_{1}$-continuous, then $F$ is fuzzy weakly $\delta-r_{1}$ continuous or $F$ is fuzzy weakly $\delta$ - $q_{1}$-continuous.

3. If $r<q$ and if $F:(\mu, \tau) \rightarrow(\nu, \sigma)$ is fuzzy weakly $\delta-r_{2}$-continuous, then $F$ is fuzzy weakly $\delta$ - $[r, q]_{2}$-continuous.

4. If $q<r$ and if $F:(\mu, \tau) \rightarrow(\nu, \sigma)$ is fuzzy weakly $\delta-[r, q]_{2}$-continuous, then $F$ is fuzzy weakly $\delta$ $r_{2}$-continuous and $F$ is fuzzy weakly $\delta$ - $q_{2}$-continuous.

Definition 12: Let $(\mu, \tau),(v, \sigma)$ be smooth fuzzy topological spaces, $F: \mu, \rightarrow v$, be a fuzzy proper function and $r, q \in I_{0}$ be fixed. Then, $F$ is called

(1) fuzzy almost $r_{1}$-continuous or $F A \delta-r_{1}-C$ if $F\left(C_{\tau}(A, r)\right) \leq \mathbb{D}_{\sigma}(F(A), r), \forall A \in \mathcal{J}_{\mu}$,

(2) fuzzy almost $[r, q]_{1}$-continuous or $F A \delta-[r, q]_{1}-C$ if

$F\left(C_{\tau}(A, r)\right) \leq \mathbb{D}_{\sigma}(F(A), q), \forall A \in \mathcal{J}_{\mu}$,

(3) fuzzy almost $r_{2}$-continuous or $F A \delta-r_{2}-C$ if $C_{\tau}\left(F^{-1}(V), r\right) \leq F^{-1}\left(\mathbb{D}_{\sigma}(V, r)\right), \forall V \in \mathcal{J}_{v}$

(4) fuzzy almost $[r, q]_{2}$-continuous or $F A \delta-[r, q]_{2}$ $C$ if

$C_{\tau}\left(F^{-1}(V), r\right) \leq F^{-1}\left(\mathbb{D}_{\sigma}(V, q)\right), \forall V \in \mathcal{J}_{v}$,

(5) fuzzy almost $r_{3}$-continuous or $F A \delta-r_{3}-C$ if $C_{\tau}\left(F^{-1}(V), r\right) \leq F^{-1}(V)$ for each $V \in \mathcal{J}_{v}$ with $V=\mathbb{D}_{\sigma}(V, r)$,

(6) fuzzy almost $r_{4}$-continuous or $F A \delta-r_{4}$-C if $C_{\tau}\left(\mu-F^{-1}(V), r\right)=\mu-F^{-1}(V) \forall V \in \mathcal{J}_{v}$ with $V=\mathbb{I}_{\sigma}(V, r)$.

Theorem 7: Let $F:(\mu, \tau) \rightarrow(v, \sigma)$ be a one-to-one fuzzy proper function with $v=F(\mu)$. If $F$ is fuzzy almost continuous, then $F$ is fuzzy almost $r_{1}$-continuous.

Since the proof of this theorem is similar to that of Theorem 4.7 in [11], we prefer to omit the details.

The statement of the above theorem is not true when $F$ is not one-to-one $F(\mu) \neq v$. The following counterexamples justify our statement.

Counterexample 6: Let $X=\{x, y\}, S=\{s, t\}$. If define $\mu\left\{\begin{array}{c}{[0.7,0.5]} \\ {[x, y]}\end{array} \in I^{X}, v_{[s, t]}^{[0.7,0]} \in I^{S}, U_{1}{ }_{[x, y]}^{[0.3,0.3]} \in \mathcal{J}_{\mu}\right.$ and $V_{1}{ }_{[s, t]}^{[0.3,0]} \in \mathcal{J}_{v}$

We define smooth fuzzy topologies $\tau$ on $\mu$ and $\sigma$ on $v$ by

$\tau(U)=\left\{\begin{array}{lc}1, & U=0_{X} \text { or } \mu \\ 0.6, & U=U_{1} \\ 0, & \text { otherwise }\end{array}\right.$ 
and

$\sigma(V)= \begin{cases}1, & V=0_{S} \text { or } v \\ 0.5, & V=V_{1}, \\ 0, & \text { otherwise. }\end{cases}$

Let the fuzzy proper function $F:(\mu, \tau) \rightarrow$ $(\nu, \sigma)$ be defined by

$$
F(x, s)=0.7, F(x, t)=0, F(y, s)=0.5, F(y, t)=0 .
$$

Then, $F$ is not one-to-one and $F(\mu)_{[s, t]}^{[0.7,0]}=v$. We fix $r=0.5$. For the $R_{\sigma}^{r}$-neighborhood $V_{1}$ of any $F\left(P_{l}^{\eta}\right)$, there exists $U_{1}$ as a $Q_{\tau}^{r}$-neighborhood of $P_{l}^{\eta}$ such that $F\left(U_{1}\right)_{[s, t]}^{[0.3,0]}=V_{1}$. For $v$, we choose $\mu$ as a $Q_{\tau}^{r}$-neighborhood $P_{l}^{\eta}$ such that $F(\mu)=v$. Hence $F$ is fuzzy almost continuous.

Since $P_{y}^{0.45} q U_{1}[\mu]$ and $P_{y}^{0.45} q \mu[\mu], U_{1}$ and $\mu$ are the $Q_{\tau}^{r}$-neighborhoods $P_{y}^{0.45}$. Clearly, we have $P_{y}^{0.45} \in C_{\tau}(A, r)$ and $F\left(P_{y}^{0.45}\right)=P_{S}^{0.45}=$ $F\left(C_{\tau}(A, r)\right)$. Since,

$$
\begin{gathered}
V_{1}(s)+0.45>0.7=v(s) \text { and } I_{\sigma}\left(C_{\sigma}\left(V_{1}, r\right), r\right)= \\
C_{\sigma}\left(\left(v-V_{1}\right)_{[s, t]}^{[0.4,0]}, r\right)=V_{1},
\end{gathered}
$$

we get that $V_{1}$ is an $R_{\sigma}^{r}$-neighborhood of $P_{S}^{0.45}$. Since $F(A){ }_{[s, t]}^{[0.3,0]} \bar{q} C_{\sigma}(V, r)[v]$, we have $P_{S}^{0.45} \notin$ $\mathbb{D}_{\sigma}(F(A), r)$ and hence $F$ is not fuzzy almost $r_{1}$ continuous.

Counterexample 7: Let $X=\{x, y\}, S=\{s, t\}$. Define the fuzzy subsets $\mu\left\{\begin{array}{c}{[0.7,0.6]} \\ {[x, y]}\end{array} \in I^{X}, v_{[s, t]}^{[0.7,0.8]} \in\right.$ $I^{S}, U_{1}{ }^{[0.3,0.3]} \in \mathcal{J}_{\mu}$, and $V_{1}{ }^{[0, y]}{ }_{[s, t]}^{[0.4]} \in \mathcal{J}_{\nu}$.

If $\tau: \mathcal{J}_{\mu} \rightarrow I$ and $\sigma: \mathcal{J}_{\nu} \rightarrow I$ are respectively, defined by

$\tau(U)=\left\{\begin{array}{lc}1, & U=0_{X} \text { or } \mu \\ 0.6, & U=U_{1} \\ 0, & \text { otherwise }\end{array}\right.$

and

$\sigma(V)= \begin{cases}1, & V=0_{S} \text { or } v \\ 0.5, & V=V_{1}, \\ 0, & \text { otherwise. }\end{cases}$

then $(\mu, \tau)$ and $(\nu, \sigma)$ are smooth fuzzy topological spaces. If $F:(\mu, \tau) \rightarrow(\nu, \sigma)$ is defined by

$$
F(x, s)=0.7, F(x, t)=0, F(y, s)=0, F(y, t)=0.6,
$$

then as in the previous counterexample, we can verify that $F$ is one-to-one, $F(\mu) \underset{[s, t]}{[0.7,0.6]} \neq v$ and $F$ is fuzzy almost continuous.

Next, we claim that $F\left(C_{\tau}(A, r)\right) \$$ $\mathbb{D}_{\sigma}(F(A), r)$, for $A_{[x, y]}^{[0,0.4]} \in \mathcal{J}_{\mu}$. Since $P_{y}^{0.41} q U_{1}[\mu]$ and $P_{y}^{0.41} q \mu[\mu]$, we get that $U_{1}$ and $\mu$ are the $Q_{\tau}^{r}$ - neighborhoods $P_{y}^{0.41}$. We have, $U_{1}(\mathrm{y})+A(\mathrm{y})>0.6$ $=\mu(\mathrm{y}), P_{y}^{0.41} \in C_{\tau}(A, r)$ and $F\left(P_{y}^{0.41}\right)=P_{t}^{0.41}=$ $F\left(C_{\tau}(A, r)\right)$.Using $V_{1}(\mathrm{t})+0.41>0.8=v(t)$ and $I_{\sigma}\left(C_{\sigma}\left(V_{1}, r\right), r\right)=V_{1}$, we obtain that $V_{1}$ is an $R_{\sigma}^{r}$ neighborhood of $P_{S}^{0.41}$. However, $F(A){ }_{[s, t]}^{[0,0.4]}$ is not quasi-coincident with $C_{\sigma}(V, r)$ in $v$. Therefore, $F$ is not fuzzy almost $r_{1}$-continuous.

Theorem 8: Let $F:(\mu, \tau) \rightarrow(v, \sigma)$ be a fuzzy proper function. If $(a) F$ is fuzzy almost $r_{1}$-continuous, $(b) F$ is fuzzy almost $r_{2}$-continuous, (c) $F$ is fuzzy almost $r_{3}$-continuous, then $(a) \Rightarrow(b) \Rightarrow(c)$.

The proof of the theorem is straightforward.

Theorem 9: Let $F:(\mu, \tau) \rightarrow(\nu, \sigma)$ be a one-to-one fuzzy proper function with $v=F(\mu)$. If $F$ is fuzzy almost $r_{3}$-continuous, then $F$ is almost $r_{4}$-continuous.

Proof. If $V \in \mathcal{J}_{v}$ is such that $V=\mathbb{I}_{\sigma}(V, r)$, then $v$ $V=v-\mathbb{I}_{\sigma}(V, r)=\mathbb{D}_{\sigma}(v-V, r)$. Using hypothesis, we get $C_{\tau}\left(F^{-1}(\nu-V), r\right)=F^{-1}(\nu-V)$. Since $F$ is one-to-one and $v=F(\mu)$, using Theorem 2, we have $F^{-1}(v-V)=\mu-F^{-1}(V)$. Therefore,

$$
C_{\tau}\left(\mu-F^{-1}(V), r\right)=\mu-F^{-1}(V) . \square
$$

The statement of the above theorem is not true when $F$ is not one-to-one or $F(\mu) \neq v$. The following counterexamples justify our statement.

Counterexample 8: Let $X=\{x, y\}, S=\{s, t\}$. We define, $\mu_{[x, y]}^{[0.8,0.6]} \in I^{X}, v_{[s, t]}^{[0.8,0]} \in I^{S}, U_{1}{ }_{[x, y]}^{[0.4,0.2]} \in \mathcal{J}_{\mu}$, $V_{1}{ }_{[s, t]}^{[0.4,0]} \in \mathcal{J}_{\nu}$.

We define $\tau: \mathcal{J}_{\mu} \rightarrow I$ and $\sigma: \mathcal{J}_{\nu} \rightarrow I$ by

$\tau(U)=\left\{\begin{array}{lc}1, & U=0_{X} \text { or } \mu \\ 0.6, & U=U_{1} \\ 0, & \text { otherwise }\end{array}\right.$

and

$\sigma(V)=\left\{\begin{array}{l}1, \quad V=0_{s} \text { or } v, \\ 0.4, \quad U=V_{1}, \\ 0, \quad \text { otherwise. }\end{array}\right.$

Let the fuzzy proper function $F:(\mu, \tau) \rightarrow$ $(\nu, \sigma)$ be defined by

$$
F(x, s)=0.8, F(x, t)=0, F(y, s)=0.6, F(y, t)=0 .
$$

We fix $r=0.4$. Since $\mathbb{D}_{\sigma}\left(V_{1}, r\right)=V_{1}$ and $\mathbb{I}_{\sigma}\left(V_{1}, r\right)=V_{1}$, we obtain that $F^{-1}\left(V_{1}\right)^{[0.4,0.4]}=$ $C_{\tau}\left(\mu-U_{1}, r\right)$. Hence, $F$ is fuzzy almost $r_{3}$-continuous. But

$$
\begin{gathered}
C_{\tau}\left(\left(\mu-F^{-1}\left(V_{1}\right)\right) \stackrel{[0.4,0.2]}{[x, y]}, r\right)=\mu-U_{1} \\
\neq U_{1}=\mu-F^{-1}\left(V_{1}\right)
\end{gathered}
$$


implies that $F$ is not fuzzy almost $r_{4}$-continuous.

Counterexample 9: Let $X=\{x, y\}, S=\{s, t\}$, $\mu_{[x, y]}^{[0.8,0.6]} \in I^{X}, v_{[s, t]}^{[1,0.8]} \in I^{S}, U_{1}{ }_{[0,3, y]}^{[0.3,0.2]}, V_{1}{ }_{[0.0, t]}^{[0.5,0.4]}$.

If $\tau: \mathcal{J}_{\mu} \rightarrow I$ and $\sigma: \mathcal{J}_{\nu} \rightarrow I$ are respectively, defined by

$\tau(U)=\left\{\begin{array}{lc}1, & U=0_{X} \text { or } \mu \\ 0.5, & U=U_{1} \\ 0, & \text { otherwise }\end{array}\right.$

and

$\sigma(V)=\left\{\begin{array}{lc}1, & V=0_{s} \text { or } v, \\ 0.4, & U=V_{1}, \\ 0, & \text { otherwise. }\end{array}\right.$

then $(\mu, \tau)$ and $(\nu, \sigma)$ are smooth fuzzy topological spaces. If $F:(\mu, \tau) \rightarrow(\nu, \sigma)$ is defined by

$$
F(x, s)=0.8, F(x, t)=0, F(y, s)=0, F(y, t)=0.6,
$$

then $F(\mu)^{[0.8,0.6]} \neq v$. We fix $r=0.4$. Since $C_{\sigma}\left(V_{1}, r\right)=V_{1}$ and $I_{\sigma}\left(V_{1}, r\right)=V_{1}$, we have $\mathbb{D}_{\sigma}\left(V_{1}, r\right)=V_{1}$. Using $F^{-1}\left(V_{1}\right)^{[0.5,0.4]}=\mu-U_{1}=$ $C_{\tau}\left(F^{-1}\left(V_{1}, r\right)\right)$, we get that $F$ is fuzzy almost $r_{3}-$ continuous. From $\mathbb{I}_{\sigma}\left(V_{1}, r\right)=V_{1}$ and $C_{\tau}(\mu-$ $\left.F^{-1}\left(V_{1}\right), r\right)=\mu-U_{1} \neq \mu-F^{-1}\left(V_{1}\right)$, we conclude that $F$ is not fuzzy almost $r_{4}$-fuzzy continuous.

The following counterexample shows that $F$ is fuzzy almost $r_{4}$-continuous but $F$ is not fuzzy almost continuous.

Counterexample 10: Let $X=\{x, y\}, S=\{s, t\}$. Define $\mu_{[x, y]}^{[0.8,0.7]} \in I^{X}, v_{[s, t]}^{[0.8,0.7]} \in I^{S}$, and $V_{1} \underset{[s, t]}{[0.4,0.3]} \in \mathcal{J}_{v}$

If $\tau: \mathcal{J}_{\mu} \rightarrow I$ and $\sigma: \mathcal{J}_{\nu} \rightarrow I$ are respectively, defined by

\begin{tabular}{|c|c|c|c|c|}
\hline & \multirow{3}{*}{$1-1, \underline{F}(\mu)=\nu$} & $\begin{array}{c}F W \delta-[r, q]_{1}-C \\
(q>r \Uparrow)(\Downarrow q<r)\end{array}$ & & $\begin{array}{c}F W \delta-[r, q]_{2}-C \\
(q>r \Uparrow)(\Downarrow q<r\end{array}$ \\
\hline $\begin{array}{l}F W \delta-C \\
\text { ix }\end{array}$ & & $F W \delta-r_{1}-C$ & $\Longrightarrow$ & $\begin{array}{c}F W \delta-r_{2}-C \\
\Downarrow\end{array}$ \\
\hline \multirow[t]{2}{*}{$F W \delta-r_{4}-C$} & & $1-1, \stackrel{F(\mu)=\nu}{\Longleftarrow}$ & & $F W \delta-r_{3}-C$ \\
\hline & & $\begin{array}{c}F A-[r, q]_{1}-C \\
(q>r \Uparrow)(\Downarrow q<r)\end{array}$ & & $\begin{array}{c}F A-[r, q]_{2}-C \\
(q>r \Uparrow)(\Downarrow q<r)\end{array}$ \\
\hline $\begin{array}{l}F A C \\
\text { FA }\end{array}$ & $\stackrel{1-1, F(\mu)=\nu}{\Longrightarrow}$ & $F A-r_{1}-C$ & $\Longrightarrow$ & $\begin{array}{c}F A-r_{2}-C \\
\Downarrow\end{array}$ \\
\hline$F A-r_{4^{-}} C$ & & $1-1, F(\mu)=\nu$ & & $F A-r_{3}-C$ \\
\hline
\end{tabular}

\section{FUZZY $q_{\alpha}^{r}$-PRE-CLOSURE AND FUZZY $q_{\alpha}^{r}$-PRE-CONTINUOUS MAPS}

Definition 13: We say that $U, V \in \mathcal{J}_{\mu}$ are said to be $\alpha$-quasi-coincident referred to $\mu$ [written as $\left.U q_{\alpha} V[\mu]\right]$ if there exists $x \in X$ such that $U(x)+V(x)$
$\tau(U)=\left\{\begin{array}{cc}1, & U=0_{X} \text { or } \mu, \\ 0, & \text { otherwise }\end{array}\right.$

and

$\sigma(V)= \begin{cases}1, & V=0_{S} \text { or } v \\ 0.5, & V=V_{1}, \\ 0, & \text { otherwise }\end{cases}$

then $(\mu, \tau)$ and $(v, \sigma)$ are smooth fuzzy topological spaces. We define a fuzzy proper function $F$ : $(\mu, \tau) \rightarrow(\nu, \sigma)$ by $F(x, s)=0.8, F(x, t)=0, F(y, s)=$ $0, F(y, t)=0.7$. If $r=0.5$, then $\mathbb{I}_{\sigma}\left(V_{1}, r\right)=0_{S}$ and hence $F$ is fuzzy almost $r_{4}$-continuous.

Clearly, $V_{1}$ is an $R_{\sigma}^{r}$-neighborhood of $F\left(P_{y}^{0.45}\right)=P_{y}^{0.45}$ and the only $Q_{\tau}^{r}$-neighborhood of $P_{y}^{0.45}$ is $\mu$. Since $F(\mu) \$ V_{1}$, we get that $F$ is not fuzzy almost continuous.

The proof of the following theorem is obvious.

Theorem 10: Let $r, q \in I_{0}$ and $F:(\mu, \tau) \rightarrow(\nu, \sigma)$.

1. If $r<q$ and if $F$ is fuzzy almost $r_{1}$-continuous, then $F$ is fuzzy almost $[r, q]_{1}$-continuous.

2. If $q<r$ and if $F:(\mu, \tau) \rightarrow(v, \sigma)$ is fuzzy almost $[r, q]_{1}$-continuous, then $F$ is fuzzy almost $r_{1}$-continuous and fuzzy almost $q_{1}$ continuous.

3. If $r<q$ and if $F:(\mu, \tau) \rightarrow(\nu, \sigma)$ is fuzzy almost $r_{2}$-continuous, then $F$ is fuzzy almost $[r, q]_{2}$-continuous.

4. If $q<r$ and if $F:(\mu, \tau) \rightarrow(\nu, \sigma)$ is fuzzy almost $[r, q]_{2}$-continuous, then $F$ is fuzzy almost $r_{2}$-continuous and $F$ is fuzzy almost $q_{2}$-continuous.

The results obtained in this section are summarized in the following implication diagram. $>\mu(x)+\alpha$. If $U$ is not $\alpha$-quasi coincident with $V$, then we write $U \bar{q}_{\alpha} V[\mu]$.

Definition 14: A fuzzy set $U \in \mathcal{J}_{\mu}$ is called a fuzzy $q_{\alpha}^{r}$-pre-neighborhood of $\alpha$ fuzzy point $P_{x}^{\lambda}$ in $\mu$ if $P_{x}^{\lambda} q \alpha U[\mu]$ and $U$ is r-preopen. 
Definition 15: A fuzzy proper function $F: \mu \rightarrow v$ is said to be fuzzy $q_{\alpha}^{r}$-pre-continuous if for every $q_{\alpha}^{r}$ pre-neighborhood $V$ of $F\left(P_{x}^{\lambda}\right)$, there exists a $q_{\alpha}^{r}$ pre-neighborhood $U$ of $P_{x}^{\lambda}$ such that $F(U) \leq V$.

Definition 16: Let $(\mu, \tau)$ be a smooth fuzzy topological space and $A \in \mathcal{J}_{\mu}$. Then the fuzzy $q_{\alpha}^{r}$-pre-closure $\operatorname{PCl}_{\alpha}(A, r)$ of $A$ is defined as follows:

$\vee\left\{P_{x}^{\lambda}: U q_{\alpha} A[\mu]\right.$

for every $q_{\alpha}^{r}$-pre-neighborhood $U$ of $\left.P_{x}^{\lambda}\right\}$.

Theorem 11: Let $(\mu, \tau)$ be a smooth fuzzy topological space. For $A, B \in \mathcal{J}_{\mu}, r \in I_{0}$ and $\alpha \in I$, this closure operator $\mathrm{PCl}_{\alpha}$ satisfies the following properties:

(1) $P C l_{\alpha}\left(0_{X}, r\right)=0_{X}$,

(2) $A \leq P C l_{\alpha}(A, r)$,

(3) $P C l_{\alpha}(A, r) \leq P C l_{\alpha}(B, r)$ if $A \leq B$,

(4) $P C l_{\alpha}(A, r) \vee P C l_{\alpha}(B, r)=P C l_{\alpha}(A \vee B, r)$,

(5) $P C l_{\alpha}(A \wedge B, r) \leq P C l_{\alpha}(A, r) \wedge P C l_{\alpha}(A \vee B, r)$,

(6) $P C l_{\alpha}\left(P C l_{\alpha}(A, r), r\right)=P C l_{\alpha}(A, r)$.

Proof.

1. Clearly, $P C l_{\alpha}\left(0_{X}, r\right)=0_{X}$.

2. Let $P_{x}^{\lambda} \in A$ and $U$ be a $q_{\alpha}^{r}$-pre-neighborhood of $P_{x}^{\lambda}$. Then, $A(x) \geq \lambda$ and $U(x)+\lambda \mu(x)+\alpha$. Therefore, $A(x)+U(x) \geq \lambda+U(x)>\mu(x)+\alpha$. Thus, $A q_{\alpha} U[\mu]$ and hence, $P_{x}^{\lambda} \in P C l_{\alpha}(A, r)$.

3. Let $A \leq B$. Let $P_{x}^{\lambda} \in P C l_{\alpha}(A, r)$ and $U$ be a $q_{\alpha^{-}}^{r}$ pre-neighborhood of $P_{x}^{\lambda}$. Then, $U q_{a} A[\mu]$. Since $U q_{\alpha} A[\mu]$ and $A \leq B$, there exists $y \in X$ such that $U(y)+B(y) \geq U(y)+A(y)>\mu(y)+\alpha$, which implies that $P_{x}^{\lambda} \in P C l_{\alpha}(B, r)$. Thus, $P C l_{\alpha}(A, r) \leq$ $P C l_{\alpha}(B, r)$.

4. From (3), we get $P C l_{\alpha}(A, r) \vee P C l_{\alpha}(B, r) \leq$ $P C l_{\alpha}(A \vee B, r)$. If $P_{x}^{\lambda} \in P C l_{\alpha}(A \vee B, r)$ and $U$ is a $q_{\alpha}^{r}$-pre-neighborhood of $P_{x}^{\lambda}$, then $U q_{\alpha}(A \vee B)[\mu]$. If $U \bar{q}_{a} A[\mu]$ and $U \bar{q}_{\alpha} B[\mu]$, then $U+A \leq \mu+\alpha$ and $U+B \leq \mu+\alpha$. Hence, $U q_{\alpha}(A \vee B)[\mu]$, which is a contradiction. Therefore, $\operatorname{PCl}_{\alpha}(A, r) \vee$ $P C l_{\alpha}(B, r)=P C l_{\alpha}(A \vee B, r)$.

5. By (3), we have $P C l_{\alpha}(A \wedge B, r) \leq P C l_{\alpha}(A, r)$ and $P C l_{\alpha}(A \wedge B, r) \leq P C l_{\alpha}(B, r)$. Thus, $P C l_{\alpha}(A \wedge B$, $r) \leq P C l_{\alpha}(A, r) \wedge P C l_{\alpha}(B, r)$.

6. Again by using (3), we get $P C l_{\alpha}(A, r) \leq P C l_{\alpha}$ $\left(P C l_{\alpha}(A, r), r\right)$. If $P_{x}^{\lambda} \in P C l_{\alpha}\left(P C l_{\alpha}(A, r), r\right)$ and $U$ is a $q_{\alpha}^{r}$-pre-neighborhood of $P_{x}^{\lambda}$, then we have $U q_{\alpha} P C l_{\alpha}(A, r)[\mu]$. Thererfore, we can find $s \in S$ such that $U(s)+P C l_{\alpha}(A, r)(s) \geq \mu(s)+\alpha$. If $\eta=$ $P C l_{\alpha}(A, r)(s)$, then $P_{s}^{\eta} q_{\alpha} U[\mu]$ and $P_{s}^{\eta} \in P C l_{\alpha}(A$, $r)$. Therefore, $U q_{\alpha} A[\mu]$ and hence $P_{x}^{\lambda} \in$ $P C l_{\alpha}(A, r)$.

The following counterexample shows that the equality does not hold in (5).
Counterexample 11: Let $X=\{x, y\}, \mu_{[x, y]}^{[0.6,0,5]} \in$ $I^{X}, U_{1} \underset{[x, y]}{[0.3,0.3]} \in \mathcal{J}_{\mu}$.

Define $\tau: \mathcal{J}_{\mu} \rightarrow I$ by $\tau(U)=\left\{\begin{array}{lc}1, & U=0_{X} \text { or } \mu \\ 0.6, & U=U_{1} \\ 0, & \text { otherwise }\end{array}\right.$

We fix $\alpha=0.1, r=0.5, A_{[x, y]}^{[0.4,0.4]}$, and $B_{[x, y]}^{[0,0.5]}$.

Case 1. $0_{X} \neq U \leq\left(\mu-U_{1}\right)_{[x, y]}^{[0.3,0.2]}$. In this case, $I_{\tau}\left(C_{\tau}(U, r), r\right)=I_{\tau}\left(\mu-U_{1}, r\right)=0_{X} \geq U$.

Case 2. $\mu \neq U \$ \mu-U_{1}$. Here,

$$
I_{\tau}\left(C_{\tau}(U, r), r\right)=I_{\tau}(\mu, r)=\mu \geq U .
$$

If $U \in \mathcal{J}_{\mu}$ is such that $U(x)>0.3$ or $U(y)>0.2$, then $U$ is $r$-pre-open. Then, the possible $q_{\alpha}^{r}$-pre-neighborhoods of $P_{y}^{0.5}$ are $K_{[x, y]}^{[l, m]}$, where $l>0.3,0.2 \geq m$ $>0.1$ and $J_{[x, y]}^{[l, m]}$, where $l \in[0,0.6], m>0.2$. The inequalities

$K(x)+A(x)>0.3+0.4=0.7=0.6+0.1=\mu(x)+\alpha$
$J(y)+A(y)>0.2+0.4=0.6=0.5+0.1=\mu(y)+\alpha$, imply that $P_{y}^{0.5} \in P C l_{\alpha}(A, r)$. Clearly, $P_{y}^{0.5} \in$ $P C l_{\alpha}(B, r)$ and hence $P_{y}^{0.5} \in \operatorname{PCl}_{\alpha}(A, r) \wedge P C l_{\alpha}(B, r)$. We note that $K_{[x, y]}^{[0.35,0.15]}$ is a $q_{\alpha}^{r}$-pre-neighborhood of $P_{y}^{0.5}$. But, $K(x)+(A \wedge B)(x)=0.35+0=0.35<$ $0.7=\mu(x)+\alpha$ and $K(y)+(A \wedge B)(y)=0.15+0.4=$ $0.55<0.6=\mu(y)+\alpha$ imply that $P_{y}^{0.5} \notin \operatorname{PCl}_{\alpha}(A \wedge B$, r).

Theorem 12: Let $F:(\mu, \tau) \rightarrow(\nu, \sigma)$ be a one -to-one fuzzy proper function with $v=F(\mu)$. If $F$ is fuzzy $q_{\alpha}^{r}$-pre-continuous, then $F\left(P C l_{\alpha}(\mathrm{A}, r)\right) \leq P C l_{\alpha}(F(A)$, $r$ ), for every $A \in \mathcal{J}_{\mu}$.

Proof. Suppose that $P_{x}^{\lambda} \in \mathcal{J}_{v}$ is such that $P_{s}^{\lambda} \notin$ $P_{C l}(F(A), r)$. Since $F(\mu)(s)=v(s) \geq \lambda>0$, there exists $x \in X$ such that $F(x, s)=\mu(x)$ and $F\left(P_{x}^{\lambda}\right)=P_{S}^{\lambda}$. On the other hand, there exists a $q_{\alpha}^{r}$-pre-neighborhood $V$ of $F\left(P_{x}^{\lambda}\right)$ such that $V q_{\alpha} F(A)[v]$. Therefore, we get $V(s)+\lambda>v(s)+\alpha$ and $V+F(A) \leq v+\alpha$. Since $F$ is $q_{\alpha}^{r}$-pre-continuous, there exists a $q_{\alpha}^{r}$-preneighborhood $U$ of $P_{x}^{\lambda}$ such that $F(U) \leq V$. Since $F$ is one-to-one and $F(\mu)=v$, we get $U(x)+A(x) \leq$ $F(U)(s)+F(A)(s) \leq V(s)+F(A)(s) \leq v(s)+\alpha=\mu(x)$ $+\alpha$. Therefore, $U \bar{q}_{\alpha} A[\mu]$ and $F\left(P_{x}^{\lambda}\right) \notin F\left(P C l_{\alpha}(A, r)\right)$. Hence, $F\left(P C l_{\alpha}(A, r)\right) \leq P C l_{\alpha}(F(A), r)$, for every $A \in$ $\mathcal{J}_{\mu}$.

The statement of the above theorem fails to be true when $F$ is not one-to-one and $F(\mu) \neq v$. The following counterexamples justify our statement. 
Counterexample 12: Let $X=\{x, y\}, S=\{s, t\}$. Define $\mu_{[x, y]}^{[0.6,0.5]} \in I^{X}, v_{[s, t]}^{[0.6,0]} \in I^{S}, U_{1}{ }_{[x, y]}^{[0.3,0.3]} \in \mathcal{J}_{\mu}$, and $V_{1}{ }_{[s, t]}^{[0.4,0]} \in \mathcal{J}_{\nu}$.

If $\tau: \mathcal{J}_{\mu} \rightarrow I$ and $\sigma: \mathcal{J}_{\nu} \rightarrow I$ are respectively, defined by

$\tau(U)=\left\{\begin{array}{lc}1, & U=0_{X} \text { or } \mu \\ 0.6, & U=U_{1} \\ 0, & \text { otherwise }\end{array}\right.$

and

$\sigma(V)=\left\{\begin{array}{cc}1, & V=0_{S} \text { or } v, \\ 0.5, & V=V_{1}, \\ 0, & \text { otherwise, }\end{array}\right.$

then $(\mu, \tau)$ and $(\nu, \sigma)$ are smooth fuzzy topological spaces. If $F:(\mu, \tau) \rightarrow(\nu, \sigma)$ is defined by

$$
F(x, s)=0.6, F(x, t)=0, F(y, s)=0.5, F(y, t)=0,
$$

then $F$ is not one-to-one and $F(\mu)_{[s, t]}^{[0.6,0]}=v$. Fix $r=$ 0.5 and $\alpha=0.1$. First, we find all $r$-preopen sets in $\mu$ and $v$. Clearly, $0_{X}, \mu$ are $r$-preopen sets.

Case 1. $0_{S} \neq U \leq\left(\mu-U_{1}\right)^{[0.3,0.2]}$. In this case, $I_{\tau}\left(C_{\tau}(U, r), r\right)=I_{\tau}\left(\mu-U_{1}, r\right)=0 \geqq U$. Hence, , each $U$ is not $r$-preopen.

Case 2. $\mu \neq U \nsubseteq\left(\mu-U_{1}\right)$. Here, $I_{\tau}\left(C_{\tau}(U, r), r\right)=I_{\tau}(\mu, r)=\mu \geq U$. Hence, $U_{[x, y]}^{[p, q]}$ is $r$-preopen, whenever $p>0.3$ or $q>0.2$. Next we find all $r$-preopen sets in $v$. Clearly, $0_{S}, v$ are $r$ preopen sets.

Case (i). $0_{S} \neq V \leq\left(v-V_{1}\right)_{[s, t]}^{[0.2,0]}$. In this case, $I_{\sigma}\left(C_{\sigma}(V, r), r\right)=I_{\sigma}\left(v-V_{1}, r\right)=0 \geqq V$. Hence, each $V$ is not $r$-preopen.

Case (ii). $\quad v \neq V \nsubseteq\left(v-V_{1}\right)$. Here, $I_{\sigma}\left(C_{\sigma}(V, r), r\right)=I_{\sigma}(v, r)=v \geq V$. Hence, $V_{[s, 0]}^{[p, q]}$ is $r$-preopen, where $p>0.2$. We claim that $F$ is $q_{\alpha^{-}}^{r}$ pre-continuous. Clearly, $v$ is a $q_{\alpha}^{r}$-pre-neighborhood of both $F\left(P_{x}^{\lambda}\right)$ and $F\left(P_{y}^{\eta}\right)$. For $v$, we choose $\mu$ as a required $q_{\alpha}^{r}$-pre-neighborhood of both $P_{x}^{\lambda}, P_{y}^{\eta}$ such that $F(\mu)=v$. Let $V_{[s, t]}^{[l, 0]}$ be a $q_{\alpha}^{r}$-pre-neighborhood of $F\left(P_{x}^{\lambda}\right)=P_{S}^{\lambda}$. Since $V$ is $r$-preopen, we have $l>0.2$.

Case (a). $0.3 \geq l>0.2$. If we choose $U_{[x, y]}^{[l, m]}$ with $l \geq$ $m>0.2$, then $U(x)+\lambda=l+\lambda=V(s)+\lambda>v(s)+$ $\alpha=0.6+0.1=\mu(x)+\alpha$. Since $U(y)=m>0.2, U$ is $r$-preopen. Therefore, $U$ is a $q_{\alpha}^{r}$-pre-neighborhood of $P_{x}^{\lambda}$ such that $F(U)_{[s, t]}^{[l, 0]}=V$.
Case (b). $l>0.3$. In this case, we choose $U_{[x, y]}^{[l, 0]}$ as a required $q_{\alpha}^{r}$-pre-neighborhood of $P_{x}^{\lambda}$ such that $F(U)_{[s, t]}^{[l, 0]}=V$.

Let $V_{[s, t]}^{[l, 0]}$ be a $q_{\alpha}^{r}$-pre-neighborhood of $F\left(P_{y}^{\eta}\right)=P_{s}^{\eta}$. Since $V$ is $r$-preopen, we have $l>0.2$. Clearly, $U_{[x, y]}^{[0, l]}$ is $r$-preopen and $U(y)+\eta=V(s)+\eta$ $>0.7>0.5+0.1=\mu(y)+\alpha$ and hence $F$ is $q_{\alpha}^{r}$-precontinuous. Now, we claim that $F\left(\operatorname{PCl}_{\alpha}(A, r)\right) \$$ $\operatorname{PCl}_{\alpha}(A, r)$ for $A_{[x, y]}^{[0.4,0.4]}$. The possible $r$-preopen sets of $P_{x}^{0.5}$ are $K_{[x, y]}^{[l, m]}$, where $0.3 \geq l>0.2$ and $m>0.2$ and $J_{[x, m]}^{[l, m]}$, where $l>0.3$ and $m \in[0,0.5]$. Since $K(y)$ $+A(y)>\mu(y)+\alpha$ and $J(x)+A(x)>\mu(x)+\alpha$, we get that $F\left(P_{x}^{0.5}\right) \in F\left(P C l_{\alpha}(A, r)\right)$. Clearly, $V_{[s, t]}^{[0.21,0]}$ is $r$ preopen and $V(s)+0.5>v(s)+\alpha$. Since $F(A)^{[0.4,0]}$, we have $V(s)+F(A)(s)<v(s)+\alpha$ and $V(t)+F(A)(t)$ $=0<v(t)+\alpha$. Therefore, $V$ is a $q_{\alpha}^{r}$-pre-neighborhood of $F\left(P_{x}^{0.5}\right)$ and $V \bar{q}_{\alpha} F(A)[v]$. Thus, $F\left(P_{x}^{0.5}\right) \notin$ $\mathrm{PCl}_{\alpha}(F(A), r)$.

Counterexample 13: Let $X=\{x, y\}, S=\{s, t\}$. Define $\mu_{[x, y]}^{[0.7,0.6]} \in I^{X}, v_{[s, t]}^{[0.8,0.8]} \in I^{S}, U_{1}{ }_{[0, y]}^{[0.4,0.3]} \in \mathcal{J}_{\mu}$, and $V_{1}{ }^{[0.5,0.5]} \in \mathcal{J}_{v}$.

Let $\tau: \mathcal{J}_{\mu} \rightarrow I$ and $\sigma: \mathcal{J}_{\nu} \rightarrow I$ be defined by

$\tau(U)=\left\{\begin{array}{lc}1, & U=0_{X} \text { or } \mu \\ 0.6, & U=U_{1} \\ 0, & \text { otherwise }\end{array}\right.$

and

$\sigma(V)= \begin{cases}1, & V=0_{S} \text { or } v, \\ 0.5, & V=V_{1}, \\ 0, & \text { otherwise },\end{cases}$

If $F:(\mu, \tau) \rightarrow(\nu, \sigma)$ is defined by

$$
F(x, s)=0.7, F(x, t)=0, F(y, s)=0, F(y, t)=0.6,
$$

then $F$ is one-to-one and $F(\mu) \underset{[s, t]}{[0.7,0.6]}=v$. We fix $r$ $=0.5$ and $\alpha=0.2$. First, we find all $r$-preopen sets in $\mu$. Clearly, $0_{X}$ and $\mu$ are $r$-preopen sets.

Case 1. $0_{S} \neq U \leq\left(\mu-U_{1}\right)^{[0.3,0.3]}$. In this case, $I_{\tau}\left(C_{\tau}(U, r), r\right)=I_{\tau}\left(\mu-U_{1}, r\right)=0 \geqq U$. Hence, each $U$ is not $r$-preopen.

Case 2. $\mu \neq U \$\left(\mu-U_{1}\right)$. Here, $I_{\tau}\left(C_{\tau}(U, r), r\right)=$ $I_{\tau}(\mu, r)=\mu \geq U$. Hence, each $U_{[x, y]}^{[p, q]}$, is $r$-preopen, whenever $p>0.3$ or $q>0.3$.

Similarly, we can verify that $0_{S}, v$ and each $V_{[s, 0]}^{[p, q]}$ is $r$-preopen, where $p>0.3, q>0.3$. We claim 
that $F$ is $q_{\alpha}^{r}$-pre-continuous. Let $V_{[s, t]}^{[l, m]}$ be a $q_{\alpha}^{r}$ pre-neighborhood of $F\left(P_{x}^{\lambda}\right)=P_{s}^{\lambda}$. Since $V_{[s, t]}^{[l, m]}$ is $r$-preopen, we have $l>0.3$ or $m>0.3$. If we choose $U_{[x, y]}^{[p, q]}$ with $p=l$ and $q=m$, then $U(x)+\lambda=l+\lambda=$ $V(s)+\lambda>v(s)+\alpha=1>0.9=\mu(x)+\alpha$. Since $U(x)$ $>0.3$ or $U(y)>0.3, U$ is $r$-preopen. Therefore, $U$ is a $q_{\alpha}^{r}$-pre-neighborhood of $P_{x}^{\lambda}$ such that $F(U)_{[s, t]}^{[l, m]} \leq V$.

Let $V_{[s, t]}^{[l, m]}$ be a $q_{\alpha}^{r}$-pre-neighborhood of $F\left(P_{y}^{\eta}\right)=P_{t}^{\eta}$. Since $V_{[s, t]}^{[l, m]}$ is $r$-preopen, $l>0.3$ or $m$ $>0.3$, we choose $U_{[x, y]}^{[p, q]}$ with $p=l$ and $q=m$ so that $U$ is a $q_{\alpha}^{r}$-pre-neighborhood of $P_{y}^{\eta}$ such that $F(U)_{[s, t]}^{[l, m]} \leq V$.

Next, we claim that $F\left(P C l_{\alpha}(A, r)\right) \$ P C l_{\alpha}(A$, $r$, for $A_{[x, y]}^{[0.6,0.5]}$. Consider $P_{y}^{0.6} \in \mu$. The possible $r$ preopen sets of $P_{y}^{0.6}$ are $K_{[x, y]}^{[l, m]}$, where $l \in[0,0.7]$ and $m>0.3$ and $J_{[x, y]}^{[l, m]}$, where $l>0.3$ and $m \in[0$, 0.6]. From the inequalities

$$
\begin{gathered}
K(y)+A(y)>0.3+0.5>\mu(y)+\alpha \\
J(x)+A(x)>0.3+0.6=0.9=\mu(x)+\alpha,
\end{gathered}
$$

we get that $P_{y}^{0.6} \in F\left(P C l_{\alpha}(A, r)\right)$. Clearly, we have $V_{[s, t]}^{[0,0.41]}$ is $r$-preopen and $V(t)+0.6>v(t)+\alpha$. Since $F(A) \underset{[0.6,0.5]}{[s, t]}$, we have $V(s)+F(A)(s)=0+0.6<v(s)$ $+\alpha$ and $V(t)+F(A)(t)<v(t)+\alpha$. Thus, $P_{y}^{0.6} \notin$ $\mathrm{PCl}_{\alpha}(F(A), \mathrm{r})$.

The following counterexample shows that the converse of Theorem 12 is not true.

Counterexample 14. Let $X=\{x, y\}, S=\{s, t\}$. Define $\mu_{[x, y]}^{[0.8,0.6]} \in I^{X}, v_{[s, t]}^{[0.8,0.6]} \in I^{S}, U_{1}{ }_{[x, y]}^{[0.5,0.3]} \in \mathcal{J}_{\mu}$, and $V_{1}{ }_{[s, t]}^{[0.4,0.3]} \in \mathcal{J}_{\nu}$.

If $\tau: \mathcal{J}_{\mu} \rightarrow I$ and $\sigma: \mathcal{J}_{\nu} \rightarrow I$ are respectively, defined by

$\tau(U)=\left\{\begin{array}{l}1, \quad U=0_{X} \text { or } \mu \\ 0.6, \quad U=U_{1} \\ 0, \quad \text { otherwise }\end{array}\right.$

and

$\sigma(V)= \begin{cases}1, & V=0_{S} \text { or } v, \\ 0.5, & V=V_{1}, \\ 0, & \text { otherwise, }\end{cases}$

Let the fuzzy proper function $F:(\mu, \tau) \rightarrow$ $(v, \sigma)$ be defined by

$$
F(x, s)=0.8, F(x, t)=0, F(y, s)=0, F(y, t)=0.6 \text {. }
$$

We note that $F$ is one-to-one and $F(\mu)^{[0.8,0.6]}=v$. Fix $r=0.5$ and $\alpha=0.2$. First, we find all $r$-preopen sets in $\mu$. Clearly, $0_{X}$ and $\mu$ are $r$-preopen sets.

Case 1. $0_{S} \neq U \leq\left(\mu-U_{1}\right)^{[0.3,0.3]}$. In this case, $I_{\tau}\left(C_{\tau}(U, r), r\right)=I_{\tau}\left(\mu-U_{1}, r\right)=0 \geqq U$. Hence, each $U$ is not $r$-preopen.

Case 2. $\mu \neq U \not\left(\mu-U_{1}\right)$. Now, $I_{\tau}\left(C_{\tau}(U, r), r\right)=$ $I_{\tau}(\mu, r)=\mu \geq U$. Hence, if $p>0.3$ or $q>0.3$, then $U_{[x, y]}^{[p, q]}$ is an $r$-preopen fuzzy set.

Next, we find all $r$-preopen sets in $v$. Clearly, $0_{S}$ and $v$ are $r$-preopen sets.

Case (i). $0_{S} \neq V \leq\left(v-V_{1}\right)^{[0.4,0.3]}$. In this case, $I_{\sigma}\left(C_{\sigma}(V, r), r\right)=I_{\sigma}\left(v-V_{1}, r\right)=V_{1}=v-V_{1} \geq$ $V$. Hence, each $V$ is $r$ - preopen.

Case (ii). $\quad v \neq V \nsubseteq\left(v-V_{1}\right)$. Here, $I_{\sigma}\left(C_{\sigma}(V, r), r\right)=I_{\sigma}(v, r)=v \geq V$. Hence, each $V_{[s, t]}^{[p, q]}$ is $r$-preopen.

Clearly, $V_{[s, t]}^{[0,0.3]}$ an is $r$-preopen fuzzy subset in $\mathcal{J}_{\mu}$ and from the inequality $V(t)+0.55=0.3+$ $0.55>0.8=v(t)+\alpha$, we have $V$ is a $q_{\alpha}^{r}$-pre-neighborhood of $F\left(P_{y}^{0.55}\right)=P_{t}^{0.55}$. Using that the $q_{\alpha}^{r}$-preneighborhoods of $P_{y}^{0.55}$ are $K_{[x, y]}^{[l, m]}$, where $l \in[0,1]$ and $m>0.3$ and $J_{[x, y]}^{[l, m]}$, where $p>0.3$ and $0.3 \geq q>$ 0.25 and $F(K)_{[x, y]}^{[l, m]} \not V, F(J)_{[x, y]}^{[l, m]} \sharp V$, we conclude that $F$ is not $q_{\alpha}^{r}$-pre-continuous. We claim that $F\left(P C l_{\alpha}(A, r)\right) \leq P C l_{\alpha}(F(A), r)$, for every $A \in \mathcal{J}_{\mu}$. Let $A_{[x, y]}^{[l, m]}$.

Case (a). $l \geq 0.7$ or $m \geq 0.5$. In this case, every $r$ preopen set $U_{[x, y]}^{[p, q]}$ is $\alpha$-quasi coincident with $A$, where $p>0.3$ or $q>0.3$. Therefore, $F\left(P C l_{\alpha}(A, r)=\right.$ $F(\mu)$. Clearly, $F(A)(s)=l \geq 0.7$ or $F(A)(t)=m \geq 0.5$. Hence, $P C l_{\alpha}(F(A), r)=v=F(\mu)=F\left(P C l_{\alpha}(A, r)\right)$.

Case (b). $l<0.7$ and $m<0.5$. Clearly, $\mathrm{A} \leq P C l_{\alpha}(A$, $r$ ). Suppose that $\lambda>A(x)=l$. We can choose a $q_{\alpha^{-}}^{r}$ pre-neighborhood $U_{[x, y]}^{[p, q]}$ of $P_{x}^{\lambda}$, where $\mu(x)-l>p$ $>\mu(x)-\lambda$ and $q>0.3$. Therefore, $U$ is $a_{\alpha}^{r}$-preneighborhood of $P_{x}^{\lambda}$ but $U \bar{q}_{\alpha} A[\mu]$. For any $\eta>A(y)$ $=m$, we can choose a $q_{\alpha}^{r}$-pre-neighborhood $W_{[x, y]}^{[p, q]}$ of $P_{y}^{\eta}$ where $\mu(y)-m>q>\mu(y)-\eta$ and $p>0.3$. Therefore, $W$ is $a_{\alpha}^{r}$-pre-neighborhood of $P_{y}^{\eta}$ but $W \bar{q}_{\alpha} A[\mu]$. Thus, $P C l_{\alpha}(A, r)=A$.

Hence, $F\left(P C l_{\alpha}(A, r)\right)=F(A) \leq P C l_{\alpha}(F(A), r)$.

\section{CONCLUSION}

Using different notions of fuzzy closure operators, we have introduced various notions of weaker 
forms of continuities such as fuzzy weakly $\delta$-continuity, fuzzy weakly $\delta$ - $r_{1}$-continuity, fuzzy weakly $\delta$ $r_{2}$-continuity, fuzzy weakly $\delta$ - $r_{3}$-continuity, etc., and inter-relations among them are obtained completely. Further, we have introduced new notion of quasi coincidence namely $\alpha$-quasi coincidence and then a fuzzy closure operator $P C l_{\alpha}$ is introduced. Using this fuzzy closure operator, $q_{\alpha}^{r}$-pre-continuous fuzzy proper function is introduced and all properties of this function are obtained.

\section{REFERENCES}

[1] M.K. Chakraborty and T.M.G. Ahsanullah, Fuzzy topology on fuzzy sets and tolerance topology, Fuzzy. Sets. Syst., 45 (1992), 103-108.

[2] C.L. Chang, Fuzzy topological spaces, J. Math. Anal. Appl., 24 (1968), 182-189.

[3] A. K. Chaudhuri and P. Das, Some results on fuzzy topology on fuzzy sets, Fuzzy. Sets. Syst., 56 (1993), $331-336$.

[4] K. C. Chattopadhyay, and S. K. Samanta, Fuzzy topology: Fuzzy closure operator, fuzzy compactness and fuzzy connectedness, Fuzzy. Sets. Syst., 54 (1993), 207-212.

[5] M. A. FathAlla and F. S. Mahmoud, Fuzzy topology on fuzzy sets, Functions with Fuzzy closed graphs, strong fuzzy closed graphs, J. Fuzzy. Math., 9 (2001), 525-533.

[6] S. Ganguly and S. Saha, A note on $\delta$-continuity and $\delta$-connected sets in fuzzy set theory, Bull. Belg. Math. Soc. Simon Stevin., 62 (1988), 127-141.

[7] I.M. Hanafy, F.S. Mahmoud and M.M. Khalaf, Fuzzy topology on fuzzy sets: fuzzy $\gamma$-continuity and fuzzy $\gamma$-retracts, Int. J. Fuzzy Log. Intell. Syst., 5 (2005), 29-34

[8] U. Höhle and A.P. Šostak, A general theory of fuzzy topological spaces, Fuzzy. Sets. Syst., 73 (1995), 131-149.

[9] C. Kalaivani and R. Roopkumar, Fuzzy proper functions and net-convergence in smooth fuzzy topological space, Ann. Fuzzy. Math. Inform., 6 (2013), $705-725$.

[10] C. Kalaivani and R. Roopkumar, Fuzzy perfect mappings and Q-Compactness in smooth fuzzy topological spaces, Fuzzy Inf. Eng., 6 (2014), 115 - 131.

[11] C. Kalaivani and R. Roopkumar, A new closure operator and some kinds of fuzzy super continuous functions on smooth fuzzy topological spaces, Ann. Fuzzy. Math. Inform., 9 (2015), 649-663.

[12] Y.C. Kim and Y.S. Kim, Fuzzy R-cluster and fuzzy R-limit points, Kangweon-Kyungki Math. J., 8 (2000), 63-72.
[13] Y.J. Kim and J.M. Ko, Fuzzy semi-regular spaces and fuzzy $\delta$-continuous functions, Int. J. Fuzzy Log. Intell. Syst., 1 (2001), 69-74.

[14] Y.C. Kim and J. M. Ko, Fuzzy G-closure operators, Commun. Korean. Math. Soc., 18 (2003), 325-340.

[15] Y.C. Kim and J.W. Park, R-fuzzy $\delta$-closure and $r$ fuzzy $\theta$-closure sets, Int. J. Fuzzy Log. Intell. Syst., 10 (2000), 557-563.

[16] T. Kubiak and A.P. Šostak, Lower set-valued fuzzy topologies, Quaest. Math., 20 (1997), 423-429.

[17] S.J. Lee and Y.S. Eoum, Fuzzy $r$-pre-semineighborhoods and fuzzy $r$-pre-semi continuous maps, Int. J. Fuzzy Log. Intell. Syst., 1 (2001), 62-68.

[18] S.J. Lee and E.P. Lee, Fuzzy $r$-preopen and fuzzy $r$-precontinuous maps, Bull. Korean. Math. Soc., 36 (1999), 91-108.

[19] S. J. Lee and E.P. Lee, Fuzzy $r$-continuous and fuzzy $r$-semicontinuous maps, Int. J. Math. Sci., 27 (2001), 53-63.

[20] F. S. Mahmoud, M. A. FathAlla and S. M. AbdEllah, Fuzzy topology on fuzzy sets: fuzzy semicontinuity and fuzzy semiseparation axioms, Appl. Math. Com., 153 (2004), 127-140.

[21] W.K. Min and C. K. Park, R-semi-generalized fuzzy continuous maps, Kangweon-Kyungki Math. J., 15 (2007), 27-37.

[22] C. K. Park, W. K. Min and M. H. Kim, Weak smooth $\alpha$-structure of smooth topological spaces, Commun. Korean Math, Soc., 19 (2004), 143-153.

[23] A.A. Ramadan, M. A. FathAlla and S.E. Abbas, Smooth fuzzy topology on fuzzy sets, J. Fuzzy Math., 10 (2002), 59-68.

[24] S.E. Rodabaugh, Categorical foundations of variable-basis fuzzy topology, in: U. Höhle, S.E. Rodabaugh (Eds.), Mathematics of fuzzy sets: Logic, topology, and measure theory, in: handbooks of fuzzy sets series, Kluwer Academic Publishers, 1999, pp. 273-388.

[25] S.E. Rodabaugh, Powerset operator foundations for Poslat fuzzy set theories and topologies, in: U. Höhle, S.E. Rodabaugh (Eds.), Mathematics of fuzzy sets: Logic, topology, and measure theory, in: the handbooks of fuzzy sets series, Kluwer Academic Publishers, 1999, pp. 91-116.

[26] R. Roopkumar and C. Kalaivani, Continuity of fuzzy proper function on Šostak's I-fuzzy topological spaces, Commun. Korean Math. Soc., 26(2) (2011), 305-320.

[27] R. Roopkumar and C. Kalaivani, Some results on fuzzy proper functions and connectedness in smooth fuzzy topological spaces, Math. Bohem., 137(3) (2012), 311-332.

[28] A.P. Šostak, On fuzzy topological structure, Rend. Circ. Matem. Palermo Ser. II., 11 (1985), 89-103. 
НЕКОИ ПОСЛАБИ ФОРМИ НА МАЗНИ ФАЗИ НЕПРЕКИНАТИ ПРЕСЛИКУВАҢА

\section{Chandran Kalaivani ${ }^{1}$, Rajakumar Roopkumar ${ }^{2 *}$}

${ }^{1}$ Институт за математика, ССН Колеџ за инженерство, Калавакам - 603 110, Индија

${ }^{2}$ Институт за математика, Универзитет Алагапа, Караикуди - 630 004, Индија

Во овој труд, воведуваме неколку поими за непрекинати фази прави пресликувања, со користење на постоечките поими за операторите фази затворач и фази внатрешност, како што се $R_{\tau}^{r}$-затворач, $R_{\tau}^{r}$ внатрешнос итн, и ги изнесуваме сите можни врски помеѓу тие типови на непрекинатости. Понатаму ги воведуваме концептите за $\alpha$-квази-коинциденија, $q_{\alpha}^{r}$-пре-околина, $q_{\alpha}^{r}$-пре-затворач и $q_{\alpha}^{r}$-пре-непрекинати пресликувања во мазни фази тополошки простори и ги испитуваме еквивалентните услови за $q_{\alpha}^{r}$-пренепрекинатост.

Клучни зборови: Фази прави преслиувања; мазна фази топологија; мазна фази непрекинатост; фази затворач; фази внатрешност 